\title{
Insulin signaling coordinately regulates cardiac size, metabolism, and contractile protein isoform expression
}

\author{
Darrell D. Belke, ${ }^{1}$ Sandrine Betuing, ${ }^{2}$ Martin J. Tuttle, ${ }^{2}$ Christophe Graveleau, ${ }^{2}$ \\ Martin E. Young, ${ }^{3}$ Mark Pham, ${ }^{2}$ Dongfang Zhang, ${ }^{4}$ Robert C. Cooksey, ${ }^{2}$ \\ Donald A. McClain, ${ }^{2}$ Sheldon E. Litwin, ${ }^{4}$ Heinrich Taegtmeyer, ${ }^{3}$ David Severson, ${ }^{1}$ \\ C. Ronald Kahn, ${ }^{5}$ and E. Dale Abel $^{2}$ \\ ${ }^{1}$ Department of Pharmacology and Therapeutics, University of Calgary, Calgary, Alberta, Canada
2Program in Human Molecular Biology and Genetics, and Division of Endocrinology, Metabolism, and Diabetes,
University of Utah, Salt Lake City, Utah, USA
${ }^{3}$ Division of Cardiology, University of Texas-Houston Medical School, Houston, Texas, USA
${ }^{4}$ Division of Cardiology, University of Utah, Salt Lake City, Utah, USA
${ }^{5}$ Joslin Diabetes Center, Harvard Medical School, Boston, Massachusetts, USA
}

Address correspondence to: E. Dale Abel, Division of Endocrinology, Metabolism, and Diabetes, and Program in Human Molecular Biology and Genetics, University of Utah School of Medicine, 15 North 2030 East, Building 533, Room 3410 B, Salt Lake City, Utah 84112, USA.

Phone: (801) 585-0727; Fax: (801) 585-0701; E-mail: dale.abel@hmbg.utah.edu.

Darrell D. Belke and Sandrine Betuing contributed equally to this work.

Received for publication August 10, 2001, and accepted in revised form January 18, 2002.

To investigate the role of insulin signaling on postnatal cardiac development, physiology, and cardiac metabolism, we generated mice with a cardiomyocyte-selective insulin receptor knockout (CIRKO) using cre/loxP recombination. Hearts of CIRKO mice were reduced in size by $20-30 \%$ due to reduced cardiomyocyte size and had persistent expression of the fetal $\beta$-myosin heavy chain isoform. In CIRKO hearts, glucose transporter 1 (GLUT1) expression was reduced by about $50 \%$, but there was a twofold increase in GLUT4 expression as well as increased rates of cardiac glucose uptake in vivo and increased glycolysis in isolated working hearts. Fatty acid oxidation rates were diminished as a result of reduced expression of enzymes that catalyze mitochondrial $\beta$-oxidation. Although basal rates of glucose oxidation were reduced, insulin unexpectedly stimulated glucose oxidation and glycogenolysis in CIRKO hearts. Cardiac performance in vivo and in isolated hearts was mildly impaired. Thus, insulin signaling plays an important developmental role in regulating postnatal cardiac size, myosin isoform expression, and the switching of cardiac substrate utilization from glucose to fatty acids. Insulin may also modulate cardiac myocyte metabolism through paracrine mechanisms by activating insulin receptors in other cell types within the heart.

J. Clin. Invest. 109:629-639 (2002). DOI:10.1172/JCI200213946.

\section{Introduction}

Insulin signaling is an important regulator of substrate metabolism in vertebrates (1) and may play a conserved role in the regulation of reproduction (2-4) and organ and body size in most eukaryotes (5-7). In the heart, insulin has direct effects on glucose transport (8), glycolysis (9), glucose oxidation (10), glycogen synthesis (11), and protein synthesis (12). Insulin may also increase cardiac contractility (13) and may have an antiapoptotic effect on cardiomyocytes (14). In vivo, many of the effects of insulin on cardiac metabolism and function are related to the systemic effects of insulin, such as increased peripheral and coronary vasodilatation $(15,16)$, increased sodium and water uptake by the kidneys (17), and changes in the delivery of substrates to the heart (18). For example, insulin's antilipolytic effect will reduce the delivery of FFAs to the heart, which, in concert with increased intracellular malonyl coenzyme A (CoA) levels, reduce fatty acid oxidation rates (19).
Diabetes is associated with profound changes in cardiac metabolism, characterized by diminished glucose utilization, diminished rates of lactate oxidation, and increased utilization of fatty acids as a metabolic substrate $(20,21)$. Diminished glucose oxidation rates in cardiomyocytes occur as early as 48 hours after the induction of diabetes by streptozotocin (22), and impaired transcription of the major cardiac glucose transporter GLUT4 is seen within 4 days after the induction of diabetes (23). Reversal of these early changes by insulin administration suggests that impaired or absent insulin signaling may play a central role in the mechanism of these alterations. Furthermore, in hyperinsulinemic animal models of type 2 diabetes such as the Zucker diabetic (fa/fa) rat, impaired insulin signal transduction in cardiac muscle (24) is also associated with diminished glucose utilization and increased fatty acid utilization in the heart (25). In all of these models it is difficult to separate the relative contribution of intrinsic defects in cardiomyocyte 
insulin signaling from potential confounding effects of altered systemic metabolism such as hyperglycemia and hyperlipidemia.

In an attempt to dissect the complex pathophysiology of insulin action in the heart, we have used cre/loxP recombination to specifically inactivate insulin signaling in cardiac myocytes in vivo in mice, while preserving insulin signaling in other cells such as endothelial cells, vascular smooth muscle cells, and liver and skeletal muscle cells $(8,26)$. In this setting, we can discern the specific roles of insulin signaling on cardiac myocyte function and metabolism without vascular or systemic metabolic defects that could confound this analysis. Likewise, the model allows for an analysis of the effect of insulin on the heart in the absence of systemic effects of insulin deficiency, or the confounding systemic effects of insulin treatment as occur in many models of diabetes. Herein we find that mice with cardiomyocyte-selective insulin receptor knockout (CIRKO) exhibit significant reduction in myocyte size, persistence of fetal patterns of myosin gene expression, and metabolic features such as increased glycolysis and decreased fatty acid oxidation that are characteristic of the immature heart.

\section{Methods}

Generation of CIRKO mice. Mice with cardiomyocyteselective ablation of the insulin receptor (CIRKO) were generated by crossing mice that were homozygous for a floxed insulin receptor allele in which loxP sites flank exon 4 of the insulin receptor gene $\left(I R^{\operatorname{lox} / l o x}\right)(26)$ with $I R^{l o x} / l o x$ transgenic mice in which cardiac-specific expression of cre recombinase was driven by the $\alpha$-myosin heavy chain promoter (8). CIRKO mice have the genotype Cre-IRlox/lox. Littermate controls have the genotype $I R^{l o x} / l o x$. Genotyping of mice was performed as previously described $(8,26)$. The Institutional Animal Care and Use Committees of the University of Utah, the Beth Israel Deaconess Medical Center, and Harvard Medical School approved all aspects of animal care and experimentation performed in this study.

Assessment of in vivo metabolism. Glucose tolerance tests were performed in awake mice after a 12-hour fast as described (8). Metabolite assays were performed in random-fed mice. Blood glucose was measured with an Elite XL glucose meter (Bayer Corp., Elkhart, Indiana, USA). Plasma insulin was measured with the Rat Insulin ELISA Kit (CrystalChem Inc., Chicago, Illinois, USA) using rat standards. Plasma FFAs were measured using the NEFA-C kit (Wako Chemicals GmbH, Neuss, Germany) with oleic acid as the standard. Plasma triglycerides were measured using the GPO-Trinder colorimetric assay kit (Sigma Chemical Co., St. Louis, Missouri, USA).

Preparation of isolated cardiomyocytes. Mice were injected with heparin (100 U intraperitoneally) 30 minutes before sacrifice and then deeply anesthetized by intraperitoneal injection of $15 \mathrm{mg}$ of chloral hydrate. The heart was rapidly excised and arrested in ice-cold buffer. The aorta was then cannulated and retrograde- ly perfused at constant pressure $(60 \mathrm{mmHg})$ for $8-10$ minutes with buffer (in $\mathrm{mM}$ ): $\mathrm{NaCl} 126 ; \mathrm{KCl} 4.4 ; \mathrm{MgCl}_{2}$ 1.0; $\mathrm{NaHCO}_{3}$ 4.0; HEPES 10.0; 2,3-butanedione monoxime 30.0; glucose 5.5; pyruvate $1.8 ; \mathrm{CaCl}_{2} 0.025$; $\mathrm{pH} 7.3$; and $0.9 \mathrm{mg} / \mathrm{ml}$ type I collagenase. The heart was then minced and myocytes dissociated by sequential washing in buffer with gradually increasing calcium concentration until a final concentration of $1 \mathrm{mM}$ was achieved. The cells were gently pelleted by centrifugation and resuspended in a modified DMEM medium. For the determination of cell size, cells were kept in suspension at $37^{\circ} \mathrm{C}$. For glucose transport assays and analysis of insulin receptor tyrosine phosphorylation, cells were plated in laminin-coated tissue culture wells. Studies commenced after waiting for 90 minutes to allow cardiomyocytes to settle and stick to the laminin.

Determination of cardiomyocyte size. Dissociated myocytes were allowed to settle onto a glass coverslip that forms the bottom of a flow-through bath that is incorporated onto the stage of an inverted microscope. Digital images of resting myocytes were recorded, and cell length, width, and area were measured on approximately 100 randomly selected myocytes from each heart (using NIH image software). The observer was blinded to the genotype of the mice from which the myocytes were obtained.

Immunoblotting. Post-nuclear membranes were prepared from cardiac muscle and immunoblotted for glucose transporter 1 (GLUT1) and GLUT4 as described (8). Insulin receptor expression was evaluated in multiple mouse tissues following homogenization and immunoblotting as described (26). For determining insulin receptor expression in isolated cardiomyocytes, cells were lysed in HES buffer (10 mM HEPES, $5 \mathrm{mM}$ EDTA, $250 \mathrm{mM}$ sucrose, $10 \mu \mathrm{g} / \mathrm{ml}$ aprotinin, and 10 $\mu \mathrm{g} / \mathrm{ml}$ leupeptin). The lysate was then centrifuged for 90 minutes at $200,000 \mathrm{~g}$ at $4^{\circ} \mathrm{C}$ and the resulting pellet resuspended. The sample was subsequently resolved on $10 \%$ SDS-PAGE gels and insulin receptor expression determined with mouse IR- $\beta$ antibody (Santa Cruz Biotechnology Inc., Santa Cruz, California, USA). For determination of insulin receptor tyrosine phosphorylation, laminin-plated isolated cardiomyocytes were stimulated with $10 \mathrm{nM}$ insulin for 5 or 10 minutes and the reaction stopped by adding $300 \mu \mathrm{l}$ of ice-cold HES buffer. Samples were prepared as for immunoblotting, and tyrosine-phosphorylated proteins were immunoprecipitated with a phosphotyrosine antibody (Santa Cruz Biotechnology Inc.) using standard protocols (26). Immunoprecipitates were then immunoblotted with the IR- $\beta$ antibody as described above. All blots were scanned and analyzed by computerized laser densitometry (Molecular Dynamics, Sunnyvale, California, USA).

Histological analysis. Hearts were rapidly excised from deeply anesthetized mice, arrested in ice-cold buffer, perfused for 1 minute with Krebs-Henseleit buffer (KHB), and subsequently perfused for 2 minutes with $10 \%$ formaldehyde in PBS. The tissues were embedded in paraffin and sectioned at $5 \mu \mathrm{m}$. Sections were stained with hematoxylin and eosin (H\&E) to assess the 
myofiber architecture and with trichrome to evaluate for differences in interstitial collagen.

Glucose uptake in isolated cardiomyocytes. Glucose transport assays were performed in triplicate in 12-well (22 $\mathrm{mm}$ diameter) laminin-coated tissue culture plates. Laminin-plated isolated cardiomyocytes were washed twice with $1 \mathrm{ml}$ of glucose-free DMEM. Then $1 \mathrm{ml}$ of glucose-free DMEM $\left(37^{\circ} \mathrm{C}\right)$ containing $0-1 \mathrm{nM}$ insulin or $0-100 \mathrm{nM}$ IGF-1, $1 \mathrm{mM}$ pyruvate, and $0.1 \%$ BSA was added. After 40 minutes, $10 \mu \mathrm{l}$ of a 2-deoxyglucose mix containing $130 \mu \mathrm{l}$ of glucose-free DMEM, $15 \mu \mathrm{l}$ of a 100 -mM 2-deoxyglucose solution, and $5 \mu \mathrm{l}$ of a $1-\mu \mathrm{Ci} / \mu \mathrm{l}$ ${ }^{3} \mathrm{H}$ 2-deoxyglucose (NEN Life Science Products Inc., Boston, Massachusetts, USA) was added. After 30 minutes, the medium was aspirated and the cells washed twice with $1 \mathrm{ml}$ of cold PBS. Cells were lysed in $500 \mu \mathrm{l}$ of $\mathrm{NaOH} 1 \mathrm{~N}$ for 20 minutes at $37^{\circ} \mathrm{C}$. A $40-\mu \mathrm{l}$ aliquot of the lysed cells was used for measuring protein content solution using a Micro BCA Protein Assay Kit (Pierce Chemical Co., Rockford, Illinois, USA). A 400- $\mu$ l aliquot of lysed cells was counted to determine the specific activity of ${ }^{3} \mathrm{H}$ 2-deoxyglucose.

Substrate metabolism in isolated working mouse hearts. Cardiac metabolism was measured in hearts isolated from 16- to 20-week-old CIRKO and age-matched control male mice. Hearts were prepared and perfused using protocols that have been extensively described (10, 21, $27,28)$. The working heart buffer was KHB containing (in mM) $118.5 \mathrm{NaCl}, 25 \mathrm{NaHCO}_{3}, 4.7 \mathrm{KCl}, 1.2 \mathrm{MgSO}_{4}$, $1.2 \mathrm{KH}_{2} \mathrm{PO}_{4}, 2.5 \mathrm{CaCl}_{2}, 0.5$ EDTA, and 11 glucose, gassed with $95 \% \mathrm{O}_{2}, 5 \% \mathrm{CO}_{2}$ and supplemented with $0.4 \mathrm{mM}$ palmitate bound to $3 \% \mathrm{BSA}$ and $11 \mathrm{mM}$ glucose. Two series of experiments were performed: insulin-free perfusions, and perfusions in which insulin was added to the working buffer to a final concentration of $1 \mathrm{nM}$. For determination of metabolism, rates of glycolysis for exogenous glucose, glucose oxidation, and palmitate oxidation were measured over a 60 -minute period in working hearts from wild-type and CIRKO mice that were perfused at a preload of 15 $\mathrm{mmHg}$, and aortic pressures and cardiac performance were determined as described $(10,21)$.

Throughout the 60-minute perfusion, pressure and flow measurements were obtained every 10 minutes. At 20 -minute intervals (starting at 0 minutes), a $2.5-\mathrm{ml}$ sample of buffer was withdrawn for determinations of metabolite content. All determinations of substrate metabolism for each time point were made in duplicate. At the end of the experiment, the hearts were quickly frozen between metal blocks cooled to $-80^{\circ} \mathrm{C}$, weighed, and stored at $-80^{\circ} \mathrm{C}$. A sample of heart tissue $(\sim 20 \mathrm{mg})$ was cut from the heart, weighed (wet weight), and then dried to constant weight (dry weight). The ratio of this sample (dry to wet weight) was used to calculate the total dry mass of the heart.

Glycolysis and glucose oxidation were measured simultaneously in one set of hearts, while palmitate oxidation was measured in a separate set of hearts. Glycolytic flux was determined by measuring the amount of ${ }^{3} \mathrm{H}_{2} \mathrm{O}$ released from the metabolism of exogenous $\left[5-{ }^{3} \mathrm{H}\right]$ glucose (specific activity $=400 \mathrm{Mbq} / \mathrm{mol}$ ). Glucose oxidation was determined by trapping and measuring ${ }^{14} \mathrm{CO}_{2}$ released by the metabolism of $\left[\mathrm{U}-{ }^{14} \mathrm{C}\right]$ glucose (specific activity $=400 \mathrm{Mbq} / \mathrm{mol}$ ). Palmitate oxidation was determined in separate perfused hearts by measuring the amount of ${ }^{3} \mathrm{H}_{2} \mathrm{O}$ released from $\left[9,10-{ }^{3} \mathrm{H}\right]$ palmitate (specific activity $=18.5 \mathrm{Gbq} / \mathrm{mol}$ ); calculation of palmitate oxidation rates took into consideration the endogenous fatty acid content of the BSA in the perfusate. Metabolic rates were calculated using the total dry mass of the heart to correct for variations in heart size.

Measurement of cardiac glucose uptake in vivo. Cardiac 2-deoxyglucose uptake was determined in awake mice using a modification of the euglycemic glucose clamp technique as previously described (29). To determine maximal insulin-stimulated glucose uptake, chronically catheterized mice were infused with insulin $(5$ $\mathrm{mU} / \mathrm{kg} / \mathrm{min}$ ) and a $50 \%$ dextrose solution to maintain euglycemia, via a variable infusion pump. To determine basal glucose uptake, mice were infused with saline alone. After documenting that blood glucose values were stable for 10 minutes, a bolus injection of 2-deoxyD- $\left[1-{ }^{3} \mathrm{H}\right]$ glucose and $\left[\mathrm{U}-{ }^{14} \mathrm{C}\right]$ sucrose $(400 \mathrm{pmol}$ of each, $11 \mathrm{Ci} / \mathrm{mmol}$ and $667 \mathrm{mCi} / \mathrm{mmol}$, respectively; Amersham Pharmacia Biotech Inc., Piscataway, New Jersey, USA) was administered. Clamps were continued for an additional 20 minutes, after which mice were euthanized with avertin and hearts and hindlimb muscles collected, weighed, and frozen in liquid nitrogen. The materials were processed and the rate of accumulation of intracellular 2-deoxyglucose determined as previously described (29).

\section{Table 1}

Primer and probe sequences used in real-time quantitative RT-PCR

\begin{tabular}{|c|c|c|}
\hline Gene & Primer/Probe & Sequence \\
\hline \multirow[t]{3}{*}{$\beta$-actin } & Forward & 5'-ССТСТGAACCCTAAGGCCAA-3' \\
\hline & Reverse & 5'-AGCCTGGATGGCTACGTACA-3' \\
\hline & Probe & 5-'FAM-TGACCCAGATCATGTTTGAGACCTTCAAC-TAMRA-3' \\
\hline \multirow[t]{3}{*}{$M C A D$} & Forward & 5'-TGGCATATGGGTGTACAGGG-3' \\
\hline & Reverse & 5'-CCAAATACTTCTTCTTCTGTTGATCA-3' \\
\hline & Probe & 5'-FAM-AGGCATTTGCCCCAAAGAATTTGCTTC-TAMRA-3' \\
\hline \multirow[t]{3}{*}{$\beta-M H C$} & Forward & 5'-AGGGCGACCTCAACGAGAT-3' \\
\hline & Reverse & 5'-CAGCAGACTCTGGAGGCTCTT-3' \\
\hline & Probe & 5'-FAM-AGCTCAGCCATGCCAACCGTATGG-TAMRA-3' \\
\hline \multirow[t]{3}{*}{ PDK4 } & Forward & $5^{\prime}$-TTCACACCTTCACCACATGC-3' \\
\hline & Reverse & 5'-AAAGGGCGGTTTTCTTGATG-3' \\
\hline & Probe & 5'-FAM-CGTGGCCCTCATGGCATTCTTG-TAMRA-3' \\
\hline \multirow[t]{3}{*}{ PPAR $\alpha$} & Forward & 5'-ACTACGGAGTTCACGCATGTG-3' \\
\hline & Reverse & 5'-TTGTCGTACACCAGCTTCAGC-3' \\
\hline & Probe & 5'-FAM-AGGCTGTAAGGGCTTCTTTCGGCG-TAMRA-3' \\
\hline
\end{tabular}


Measurement of tissue glycogen and triglyceride content. Glycogen content was measured in 10-20 mg of heart tissue using a modification of the protocol of Chan and Exton (30). Samples were dissolved in $0.3 \mathrm{ml} 0.5 \mathrm{~N}$ $\mathrm{KOH}$ and incubated at $95^{\circ} \mathrm{C}$ for 30 minutes. The glycogen, which was precipitated in $\mathrm{Na}_{2} \mathrm{SO}_{4}$ and methanol, was then digested with amyloglucosidase (Sigma Chemical Co.), and the glucose produced was determined by using the Glucose (Trinder) assay kit from Sigma Chemical Co. Triglyceride content in cardiac tissue was measured after chloroform/ethanol extraction as described (31).

RNA extraction and quantitative RT-PCR. RNA extraction and quantitative RT-PCR of samples were performed as described $(32,33)$. Specific quantitative assays were designed from mouse sequences available in GenBank (Table 1). Primers and probes were designed from nonconserved sequences of the genes (allowing for isoform specificity), spanning sites where two exons join (splice sites) when such sites were known, thus preventing recognition of the assay to any potential contaminating genomic DNA. Standard RNA was made for all assays by the T7 polymerase method (Ambion Inc., Austin, Texas, USA), using total RNA isolated from the mouse heart. The correlation between the $C_{t}$ (the number of PCR cycles required for the fluorescent signal to reach a detection threshold) and the amount of standard was linear over at least a 5 -log range of RNA for all assays (data not shown). Transcript levels for the constitutive housekeeping gene product $\beta$-actin were quantitatively measured in each sample, and PCR data are reported as the number of transcripts per number of $\beta$-actin molecules. Northern blotting was also performed as described (8) using species-specific cDNA probes (kindly provided by Daniel Kelly, Washington University School of Medicine, St. Louis, Missouri, USA) to validate the results of the RT-PCR and to determine the expression levels of carnitine palmitoyl transferase-1 (CPT-1) and medium-, long-, and very-long-chain acyl CoA dehydrogenases (MCAD, LCAD, and VLCAD, respectively). Blots were probed with GAPDH cDNA (Ambion Inc.) to correct for loading. Filters were exposed to a PhosphorImager cassette and transcript abundance quantified by a PhosphorImager (Molecular Dynamics).

Mouse echocardiography. Transthoracic echocardiography was performed in mice lightly anesthetized with avertin $(0.2 \mathrm{ml} / 10 \mathrm{~g}$ body weight). Heart rates are generally maintained at more than 400 per minute with this regimen. The chest hair was removed with a topical depilatory agent. Limb leads were attached for electrocardiogram gating, and the animals were imaged in the supine position with a 13-MHz linear probe (Vivid FiVe; GE Medical Systems, Milwaukee, Wisconsin, USA).Two-dimensional guided M-mode images were taken in both short and long axis projections. Left ventricular (LV) cavity size and wall thickness were measured in at least three beats from each projection and averaged. LV mass was calculated according to a standard cube formula (34). LV systolic function was assessed by calculating fractional shortening and ejection fraction.

Statistical analysis. Data are expressed as mean \pm SEM. Differences in glucose transporter expression, glucose uptake in isolated cardiomyocytes and in vivo, cardiac substrate metabolism, cardiac weights, and cardiac performance were analyzed by ANOVA, and significance was assessed by Fisher's protected least significant difference test. Differences in myocyte size and mRNA transcript levels were compared by the unpaired two-tailed $t$ test. Statistical calculations were performed using the Statview 5.0.1 software package (SAS Institute Inc., Cary, North Carolina, USA).

\section{Results}

Evidence for cardiac-selective deletion of the insulin receptor. CIRKO mice were born with the expected mendelian frequency and survived to adulthood. Life expectancy, growth rates, and systemic metabolism were normal. Intraperitoneal glucose tolerance and fed serum concentrations of insulin, FFAs, and triglycerides were the same in CIRKO and age-matched control mice studied at 2 and 6 months of age (data not shown). Western blotting of whole heart homogenates revealed a faint insulin receptor signal in CIRKO mice as compared with wild-type controls, while insulin receptor protein could not be detected in blots of isolated cardiomyocytes (Figure 1). This observation supports the notion that the faint band seen in cardiac homogenates originated in cell types other than cardiac myocytes. The absence of insulin receptor autophosphorylation in in vitro insulin-stimulated isolated cardiomyocytes confirmed the absence of insulin receptors in CIRKO cardiomyocytes (Figure 1).

Impact of absent insulin receptor signaling on cardiac size and structure. On gross inspection, the hearts of CIRKO mice were reduced in size (Figure 2). At 12 weeks of age, heart weight/body weight ratios were reduced by $22 \%$ and $28 \%$ in female and male CIRKO mice, respectively (Table 2). There was a uniform reduction in cardiomyocyte size (Figure 2). Myocyte length and width were reduced by $6 \%$ and $9 \%$, respectively, resulting in a $12 \%$ decrease in myocyte area. If myocytes are assumed to be cylindrical, then the change in myocyte size observed would produce a $21 \%$ decrease in cardiomyocyte volume in CIRKO mice, relative to controls. Thus, the

\section{Table 2}

Heart weights of 12-week-old CIRKO mice

\begin{tabular}{lcccc}
\hline & \multicolumn{2}{c}{ Males } & \multicolumn{2}{c}{ Females } \\
& Wild-type & CIRKO & Wild-type & CIRKO \\
Heart weight $(\mathrm{mg})$ & $106 \pm 5^{\mathrm{A}}$ & $85 \pm 8$ & $93 \pm 3^{\mathrm{A}}$ & $75 \pm 4$ \\
Heart weight/Body weight ratio & $4.31 \pm 0.09^{\mathrm{B}}$ & $3.10 \pm 0.07$ & $3.90 \pm 0.08^{\mathrm{B}}$ & $3.07 \pm 0.21$
\end{tabular}

${ }^{A} P<0.04,{ }^{B} P<0.0002$ vs. CIRKO of same sex (ANOVA). $n=5$ for all groups. 


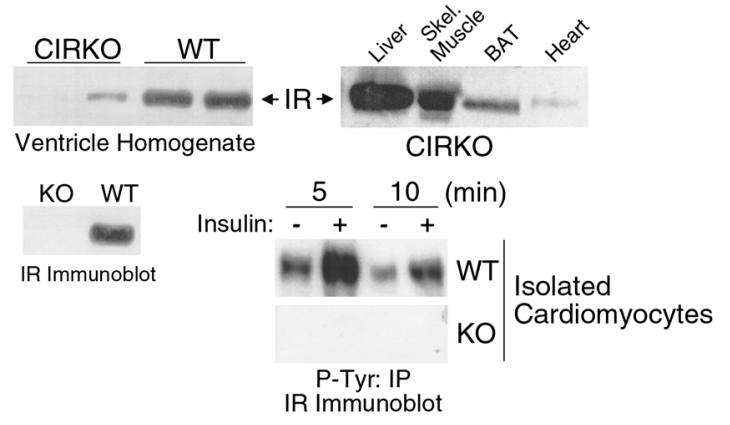

Figure 1

Insulin receptor (IR) levels and IR autophosphorylation in CIRKO mice. Upper panels show representative immunoblots blotted for the $I R$ in various tissues of CIRKO mice (right), and homogenates of cardiac ventricles from CIRKO and wild-type (WT) mice (left). Lower panels show a representative immunoblot for the IR from isolated cardiomyocytes obtained from CIRKO (KO) and WT mice (left), and a phosphotyrosine (P-Tyr) immunoprecipitate showing IR phosphorylation in cardiomyocytes from WT and $\mathrm{KO}$ mice following insulin stimulation (right). Data are from 8- to 12-week-old male mice and are representative of three to four experiments on separate animals. BAT, brown adipose tissue.

reduction in myocyte size alone may be sufficient to explain the lower cardiac mass in CIRKO mice, although a decrease in cell number cannot be completely ruled out. With the exception of the decrease in myocyte size, histological evaluation of CIRKO hearts (Figure 3) was otherwise normal, and there was no discernible difference in cardiac collagen content as assessed by trichrome staining.

Impact of absent insulin receptor signaling on glucose transport in isolated cardiac myocytes. In CIRKO mice, GLUT1 expression in the heart was reduced by $40-50 \%$ relative to controls (Figure 4) and basal glucose uptake in isolated cardiac myocytes from CIRKO was reduced in parallel (Figure 5), despite increased GLUT4. The twofold increase in GLUT4 (Figure 4) was unexpected. Physiological concentrations of insulin $(0.1 \mathrm{nM})$ caused no change in glucose uptake in CIRKO myocytes but stimulated a 3.2-fold increase in glucose uptake in wildtype cardiac myocytes. An insulin concentration of 1.0 $\mathrm{nM}$ increased glucose uptake further (4.4-fold over basal) in wild-type myocytes, and in CIRKO myocytes produced a small (37\%) statistically significant increase in glucose uptake (Figure 5). IGF-1 increased glucose uptake in wild-type cardiac myocytes 1.9- and 4.4-fold at 10 and $100 \mathrm{nM}$, respectively. In CIRKO cardiac myocytes, IGF-1 increased glucose uptake 1.8- and 8.3fold at 1 and $10 \mathrm{nM}$, respectively. Absolute rates of glucose uptake at $10 \mathrm{nM}$ were similar in CIRKO and wildtype myocytes. There was no additional increase in glucose uptake in CIRKO myocytes treated with 100 nM IGF-1, which may reflect the possibility that at this concentration, IGF-1 signals via insulin receptors, which are absent in CIRKO cardiomyocytes.

Impact of absent insulin receptor signaling on substrate metabolism in isolated working hearts and on cardiac glucose uptake in vivo. To more fully characterize the role of insulin signaling in regulating myocardial metabolism, rates of glycolysis, glucose oxidation, and palmitate oxidation and tissue content of glycogen and triglyceride were measured in isolated working hearts of CIRKO and control mice perfused with or without $1 \mathrm{nM}$ insulin. In wild-type hearts, insulin increased glycolytic rates from $3,470 \pm 338$ to $4,915 \pm 477 \mathrm{nmol} / \mathrm{min} / \mathrm{g}$ dry weight $(P<0.05)$. In CIRKO hearts, basal rates of glycolysis $(4,958 \pm 187 \mathrm{nmol} / \mathrm{min} / \mathrm{g}$ dry weight) were significantly higher than in wild-type mice and were similar to the glycolytic rates in insulin-stimulated wild-type hearts. Glycolytic rates in CIRKO hearts did not increase further when the hearts were treated with insulin (Figure 6). Glucose uptake in vivo paralleled the changes in glycolysis that were observed in isolated working hearts. In wild-type mice, insulin administration resulted in a $46 \%$ increase in cardiac glucose uptake $(P<0.04)$. In CIRKO mice, basal rates of cardiac glucose uptake were $68 \%$ percent higher than in wildtype $(P<0.005)$ and did not change further with insulin administration (Figure 6).

In wild-type hearts, insulin increased glucose oxidation rates by $24 \%$ from $1551 \pm 112$ to $1927 \pm 226 \mathrm{nmol} / \mathrm{min} / \mathrm{g}$ dry weight $(P<0.05)$. In CIRKO hearts, basal rates of glucose oxidation were $30 \%$ lower than in wild-type hearts $(P<0.05)$, but, surprisingly, insulin increased glucose oxidation rates by $63 \%$ from $1115 \pm 226$ to $1824 \pm 426$ $\mathrm{nmol} / \mathrm{min} / \mathrm{g}$ dry weight $(P<0.05)$, a rate similar to that observed in insulin-treated wild-type hearts (Figure 6).

In wild-type hearts, insulin decreased palmitate oxidation rates by $46 \%$ from $703 \pm 232$ to $382 \pm 46$ $\mathrm{nmol} / \mathrm{min} / \mathrm{g}$ dry weight $(P<0.05)$. In CIRKO hearts, basal rates of palmitate oxidation were $34 \%$ lower than basal rates in wild-type $(P<0.05)$ and did not change further after insulin stimulation. To investigate the possibility that differences in the utilization of endogenous triglycerides could account for the decreased dependence of CIRKO hearts on exogenous long-chain fatty acid substrates, cardiac tissue levels of triglycerides in hearts of random-fed CIRKO mice were meas-
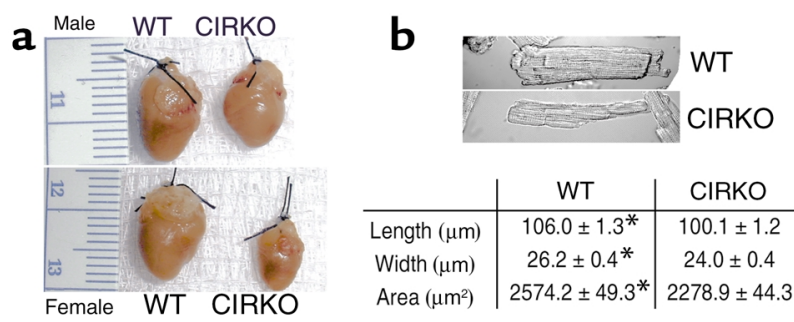

\section{Figure 2}

Heart and myocyte size in CIRKO and WT mice. (a) Examples of hearts from 5-week-old male and female CIRKO mice and littermate controls. Ventricular wall thicknesses of the hearts shown are: male WT, $2000 \mu \mathrm{m}$; male CIRKO, $800 \mu \mathrm{m}$; female WT, $1300 \mu \mathrm{m}$; and female CIRKO, $800 \mu \mathrm{m}$. (b) Representative photomicrographs of isolated cardiomyocytes obtained from 12-week-old male CIRKO mice. Myocyte dimensions ( $n=100$ myocytes/mouse $\times 3$ mice) are shown in the table below. Data are means \pm SE. ${ }^{*} P<0.0001$ vs. CIRKO. 


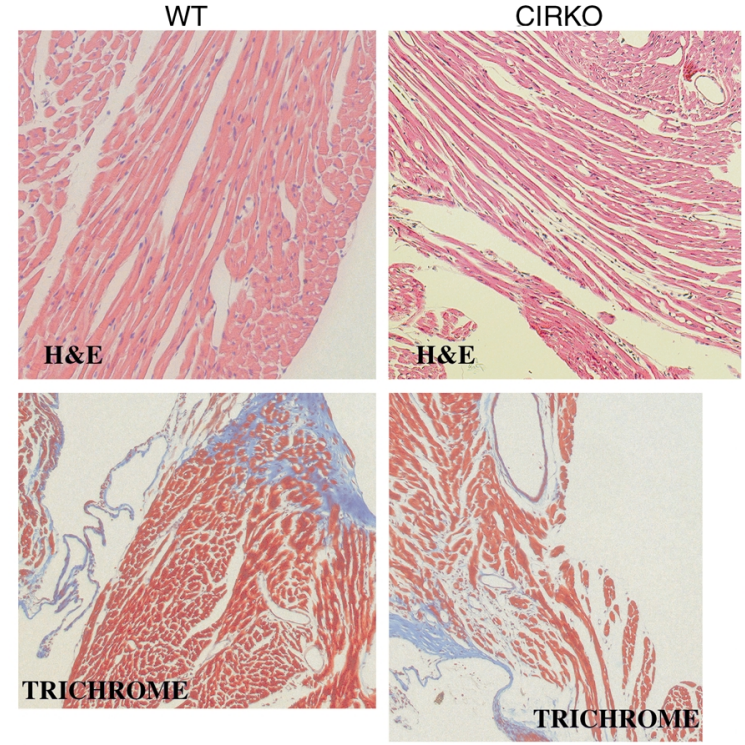

Figure 3

Cardiac histology. Representative transverse sections of left ventricle stained with $\mathrm{H} \& \mathrm{E}(\times 20$, upper panels) and Trichrome $(\times 10$, lower panels) obtained from CIRKO and WT mice. Upper panels are from 5-week-old males, and lower panels are from 5-week-old females. Both H\&E and Trichrome data exist for each sex.

ured immediately postmortem. There was no difference in cardiac triglyceride content between wild-type and CIRKO mice $(2.83 \pm 0.34$ vs. $2.98 \pm 0.45 \mu \mathrm{g}$ lipid/mg heart weight, respectively).

Glycogen content in wild-type hearts perfused in the presence and absence of insulin were also similar. CIRKO hearts perfused without insulin had 2.3 times the glycogen content of wild-type hearts $(P<0.05)$. Insulin administration reduced glycogen levels to those seen in the wild-type (Figure 6).

Cardiac function. Cardiac performance was determined concurrently with metabolic measurements in isolated hearts and is summarized in Table 3. Cardiac output was about $36 \%$ lower in CIRKO mice. After adjusting for heart weight, the difference was only $15 \%$, and this remained statistically significant only in the insulin-treated cohort. Similarly, cardiac power, which is determined by cardiac output and developed pressure, was modestly reduced in CIRKO mice. Echocardiographic assessment of CIRKO and wildtype mice is summarized in Table 4. CIRKO mice exhibited an increase in LV systolic dimension without any change in diastolic dimension. Thus, fractional shortening and ejection fraction were reduced by $29 \%$ and $12 \%$, respectively, in CIRKO mice relative to age matched controls $(P<0.02)$.

Impact of absent insulin signaling on the expression levels of genes that regulate substrate metabolism and energy utilization. To gain additional insight into the molecular mechanisms responsible for the metabolic phenotype of CIRKO hearts, the steady-state expression levels of CPT-1, and the enzymes (MCAD, LCAD, and VLCAD) that are involved in catalyzing the first step in mitochondrial fatty acid $\beta$-oxidation (35), and one of their transcriptional regulators PPAR $\alpha$ (36) were determined. In addition, expression levels of pyruvate dehydrogenase kinase 4 (PDK4), which is an important negative regulator of pyruvate dehydrogenase (PDH) (37), were determined (Figure 7). In CIRKO hearts, MCAD expression and VLCAD expression were reduced by $40 \%$ and LCAD expression by $25 \%(P<0.05)$, and there was a trend toward decreased expression of PPAR $\alpha$ (26\% reduction) relative to wild-type hearts. CPT-1 expression was unchanged (data not shown). PDK4 expression was slightly reduced in CIRKO mice (by $26 \%, P=0.2$ ), rendering it unlikely that PDK4 is involved in the mechanism for reduced glucose oxidation rates in non-insulin-stimulated CIRKO hearts. To explore the hypothesis that altered metabolic signaling by insulin may play a role in the developmental switch from $\beta$-myosin heavy chain ( $\beta$-MHC), with lower intrinsic ATPase activity, to $\alpha$-MHC, with higher intrinsic ATPase activity, that occurs shortly after birth (38), expression levels of $\beta$ isoforms of the MHC genes were determined. In CIRKO hearts, $\beta$-MHC expression was fourfold higher than in controls (Figure 7).

\section{Discussion}

By deleting insulin receptor expression in the early postnatal heart, we have discovered an important role for insulin signaling in regulating the developmental changes that characterize the transition from the immature to the mature heart. Specifically, postnatal cardiac growth is reduced, and there is persistence of a fetal pattern of cardiac metabolism characterized by increased glycolysis, reduced rates of fatty acid oxidation, and persistent expression of the fetal myosin isoform $\beta$-MHC. Furthermore, we provide evidence sug-
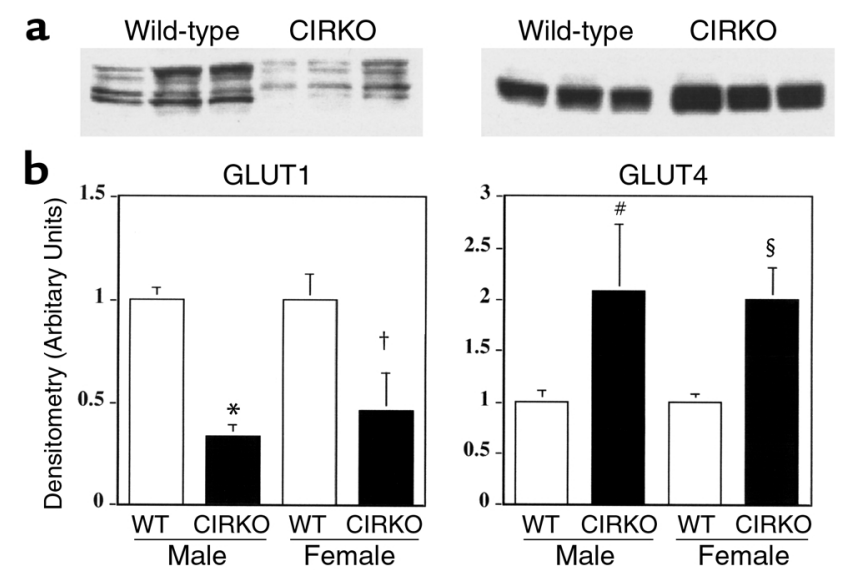

Figure 4

Glucose transporter expression. Upper panels show representative GLUT1 and GLUT4 immunoblots obtained from the hearts of 12-week-old CIRKO and littermate control mice (WT). Lower panels show densitometric analyses of six to nine independent blots. ${ }^{*} P<0.0001,+P<0.04,{ }^{\#} P<0.05$, and $\$ P<0.01$ vs. WT of the same sex. Data are means $\pm \mathrm{SE}$. 

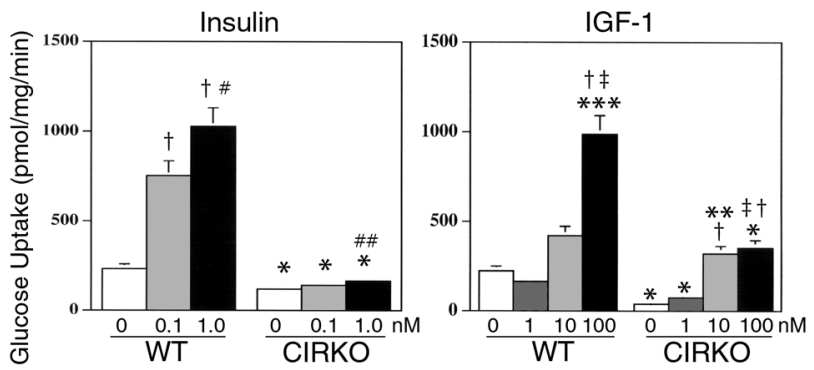

Figure 5

Glucose transport in isolated cardiomyocytes. Left panel shows 2-deoxyglucose uptake in response to insulin stimulation at the concentrations shown. Right panel shows 2-deoxyglucose uptake in response to IGF-1 stimulation at the concentrations shown. Data are obtained from cardiomyocytes obtained from 12-week-old male CIRKO and age-matched controls and represent three independent experiments performed in triplicate. Data are means \pm SE. ${ }^{*} P<0.0001$ vs. WT treated with equivalent dose of insulin or IGF-1, ${ }^{*} P<0.004$ vs. 1 nM IGF- $1,{ }^{* *} P<0.0005$ vs. 10 nM IGF- 1 , $\dagger P<0.0001$ vs. basal of same genotype, $\ddagger P<0.001$ vs. $1 \mathrm{nM}$ IGF- 1 , ${ }^{\#} P<0.002$ vs. $0.1 \mathrm{nM}$ insulin, ${ }^{\# \#} P<0.01$ vs. basal of same genotype.

gesting that insulin may regulate metabolism in cardiac muscle through paracrine mechanisms.

Much is known about the signaling mechanisms that mediate "pathological" cardiac hypertrophy in response to environmental stimuli (39). Less is known about the factors that regulate normal adult cardiac size. Cell division in the heart ceases shortly after birth, and the postnatal heart increases its size by hypertrophy of existing cardiomyocytes (40). Although IGF-1 signaling may regulate cardiac size by controlling myocyte number (41), the present study strongly suggests that the insulin-signaling pathway may be a key regulator of postnatal cardiac growth. Thus, in CIRKO mice, heart weight/body weight ratios were reduced by $22-28 \%$, and this appears to be due to a decrease in myocyte size rather than number. The role of insulin in cardiomyocyte cell size regulation is supported by studies of Drosophila with disrupted insulin signaling (6) and of mice with targeted deletion of insulin receptor substrate-1 (IRS-1) or IRS-2 $(7,42)$, which indicate conserved roles for insulin signaling in the determination of organ size. This effect may be mediated by phosphoinositide $3 \mathrm{OH}$-kinase (PI3K), a downstream mediator of many receptor tyrosine kinases including the insulin and IGF-1 receptors. Inhibition of PI3K function in the hearts of mice using a dominant negative transgene results in a phenotype that is similar in certain respects to that of CIRKO mice (43). Dominant negative PI3K transgenic mice have heart weight/body weight ratios that are $16 \%$ less than those of controls, and myocyte surface areas that are reduced by $18 \%$. Thus, impaired PI3K activity could represent one mechanism for the cardiac size phenotype in CIRKO mice.

Our data suggest that insulin signaling may play an important role in the metabolic switch from predominant glucose metabolism, as seen in neonatal hearts, to fatty acid metabolism that characterizes the adult heart. One mechanism for this transition is increased expression of genes that regulate the rate of fatty acid oxidation, mediated in part by PPAR $\alpha$, a positive transcriptional regulator of these genes (36). We observed that the expression levels of MCAD, LCAD, and VLCAD were significantly reduced in the hearts of CIRKO mice, but that expression levels of CPT-1 were unchanged.

\section{Figure 6}

Cardiac metabolism in isolated working hearts, and in vivo glucose uptake in CIRKO and control mice. Average rates of glycolysis, glucose oxidation, fatty acid oxidation, and glycogen content after 60 minutes of perfusion and in vivo cardiac 2-deoxyglucose uptake are shown in the panels as labeled. All studies were performed in 16- to 20week-old male CIRKO mice and littermate controls. In the isolated heart studies, equal numbers of CIRKO and control mice were studied in each experiment. Numbers of animals studied are as follows. Without insulin: glucose oxidation and glycolysis, $n=5$; fatty acid oxidation, $n=4$; glycogen, $n=9$. With insulin: glucose oxidation and glycolysis, $n=7$; fatty acid oxidation, $n=6$; glycogen, $n=13$. For the in vivo 2-deoxyglucose uptake studies, numbers of mice are $n=4$ and 5 (WT, basal and insulin, respectively) and $n=4$ and 3 (CIRKO, basal and insulin, respectively). Data are means $\pm \mathrm{SE}$. ${ }^{*} P<0.05$ vs. basal, ${ }^{\dagger} P<0.05$ vs. WT.
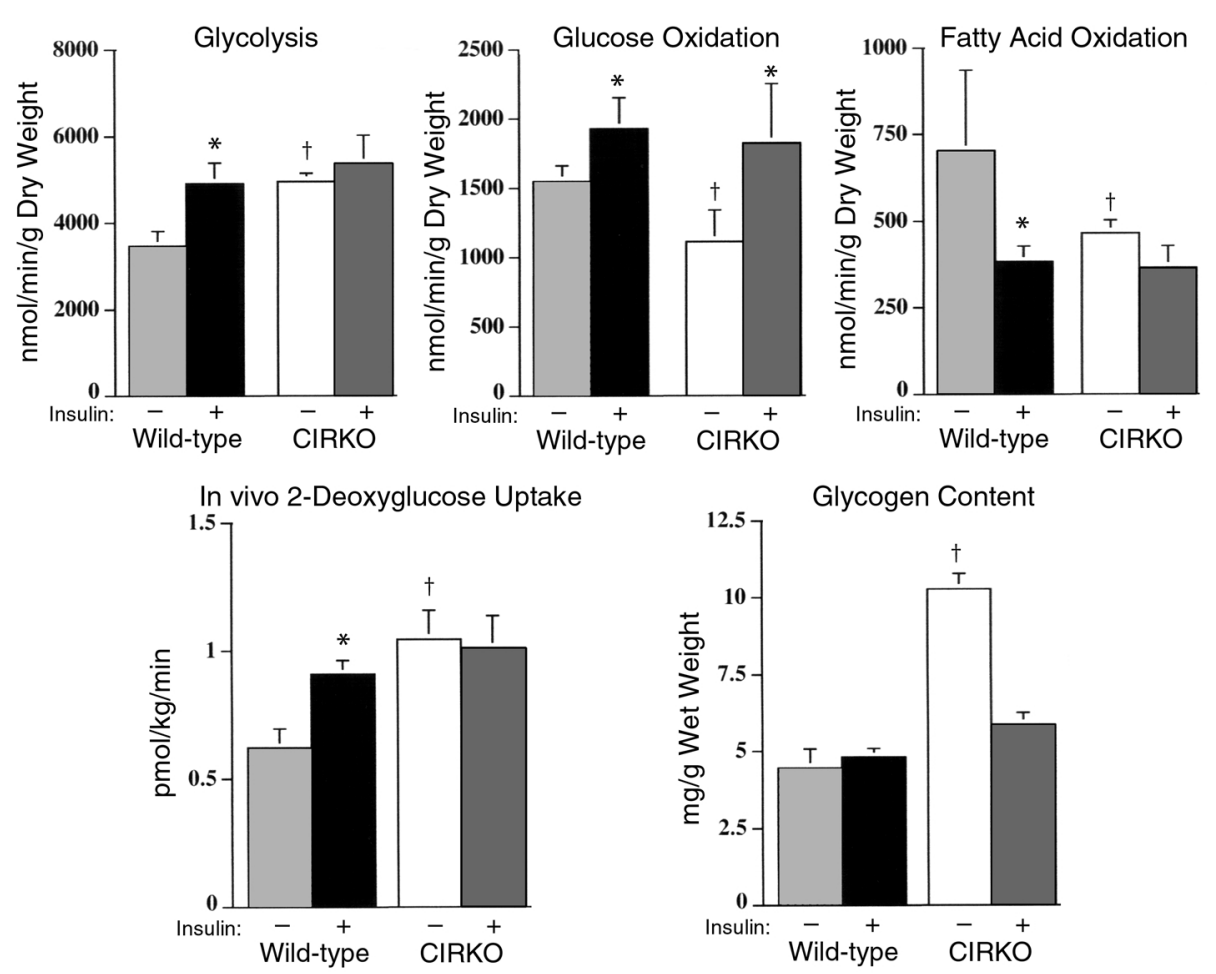

Glycogen Content

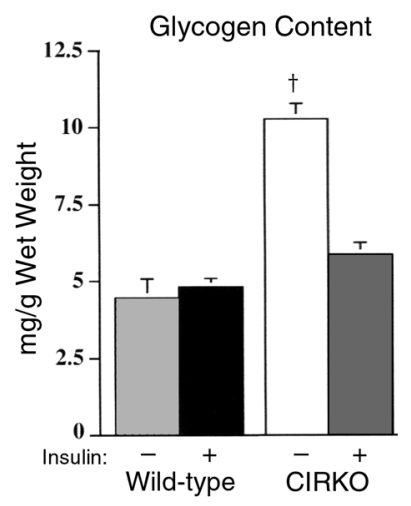


Mean cardiac performance (during 60-minute perfusion) in isolated working wild-type and CIRKO hearts

\begin{tabular}{|c|c|c|c|c|}
\hline & \multicolumn{2}{|c|}{ - Insulin } & \multicolumn{2}{|c|}{$+1 \mathrm{nM}$ insulin } \\
\hline & Wild-type (9) & CIRKO (9) & Wild-type (14) & CIRKO (13) \\
\hline Ventricular weight (HW) (mg) & $135 \pm 2^{\mathrm{D}}$ & $97 \pm 1$ & $147.2 \pm 2.1^{\mathrm{D}}$ & $112.5 \pm 1.5$ \\
\hline Cardiac output (CO) ( $\mathrm{ml} / \mathrm{min})$ & $7.2 \pm 0.5^{\mathrm{D}}$ & $4.6 \pm 0.3$ & $7.8 \pm 0.2^{\mathrm{D}}$ & $5.1 \pm 0.4$ \\
\hline $\mathrm{CO} / \mathrm{HW}(\mathrm{ml} / \mathrm{min} / \mathrm{mg})$ & $0.055 \pm 0.004$ & $0.047 \pm 0.004$ & $0.055 \pm 0.002^{\mathrm{A}}$ & $0.047 \pm 0.003$ \\
\hline Coronary flow (CF) (ml/min) & $2.8 \pm 0.1^{\mathrm{C}}$ & $2.3 \pm 0.1$ & $2.5 \pm 0.1$ & $2.4 \pm 0.1$ \\
\hline $\mathrm{CF} / \mathrm{HW}(\mathrm{ml} / \mathrm{min} / \mathrm{mg})$ & $0.021 \pm 0.001$ & $0.023 \pm 0.001$ & $0.017 \pm 0.001^{\mathrm{D}}$ & $0.022 \pm 0.001$ \\
\hline Cardiac power ( $\mathrm{mW} / \mathrm{g}$ dry heart weight) & $58.3 \pm 3.9^{\mathrm{A}}$ & $46.7 \pm 3.1$ & $44.8 \pm 1.8^{\mathrm{B}}$ & $37.2 \pm 2.3$ \\
\hline
\end{tabular}

PPAR $\alpha$ expression was only marginally reduced. Taken together, the normal expression of CPT- 1 and the marginal change in PPAR $\alpha$ suggest that alternative transcriptional pathways may be involved in the ability of insulin to regulate the expression of genes involved in regulating mitochondrial $\beta$-oxidation. Transcription of the MCAD gene is negatively regulated by Sp1 and COUP-TF (44). Activation of Sp1, which is a glucosesensing transcription factor (45), could potentially contribute to the repression of MCAD expression and the induction of $\beta$-MHC expression in CIRKO mice. $\beta$-MHC expression, which is classically induced during pressure overload cardiac hypertrophy (46), is also increased by cardiac atrophy (as develops in heterotopically transplanted hearts) (33) and in rats with type 1 diabetes (47). A unifying mechanism in all of these models is discordance between glycolytic flux and glucose oxidation. This results in accumulation of glucose metabolites (such as products of the hexosamine biosynthetic pathway) that glycosylate and activate glucose-sensing transcription factors such as Sp1, which in turn increases $\beta$-MHC transcription (44). Indeed, carbohydrate restriction prevents the induction of $\beta$-MHC in rats with pressure overload hypertrophy (48). The possibility therefore exists that a critical mechanism that is operational in CIRKO mice is the upregulation of glucose transport (via increased GLUT4 expression), which in turn leads to increased glycolysis that may secondarily trigger fetal programs. Therefore, it will be important in future studies to analyze Sp1 and other glucose-sensing transcription factors in the CIRKO mouse to determine whether these transcriptional pathways are central to the developmental regulation of myosin isoform expression and substrate utilization in the heart by insulin.

Insulin suppresses fatty acid oxidation rates in normal hearts. This is mediated in part by the ability of insulin to increase glucose utilization. Increased glucose metabolism leads to an increase in intracellular malonyl $\mathrm{CoA}$, which in turn inhibits fatty acid oxidation via reversal of the Randle's cycle (49). Intracellular concentrations of malonyl CoA were not measured in the present study, but, given the diminished rates of glucose oxidation in CIRKO hearts perfused in the absence of insulin, a decrease in malonyl CoA concentrations would be expected, which would in turn be associated with increased rates of fatty acid oxidation. Thus it could be expected that fatty acid oxidation rates may increase in the absence of insulin signaling in the heart. In contrast, despite lower basal rates of glucose oxidation, fatty acid oxidation in CIRKO hearts was depressed. Thus, in addition to its acute effect in regulating cardiac fatty acid metabolism, insulin also plays an important role in regulating the basal expression of mitochondrial $\beta$-oxidation enzymes that in turn control fatty acid metabolic flux in the heart.

The CIRKO mouse also provided novel insight into the regulation of glucose transporter expression by insulin and the relative roles of GLUT1 and GLUT4 in mediating basal cardiac glucose uptake. Diabetes is associated with reduced levels of GLUT1 and GLUT4 in the heart $(50,51)$. Forty-eight hours of fasting in rats also leads to downregulation of cardiac GLUT1 expression (52), whereas euglycemic hyperinsulinemia leads to upregulation (53). Activation of the ras-mitogen-activated protein kinase (ras-MAPK) pathway leads to increased GLUT1 transcription in vitro (54). Thus, our findings of reduced GLUT1 expression in CIRKO hearts support prior studies that point to an important role for insulin signaling in the regulation of cardiac GLUT1 expression, and they suggest that deficiency of insulin or insulin action may be an important mechanism for reduced GLUT1 expression in the myocardium in diabetes. Acute insulin deficiency results in a rapid reduction in the expression of a GLUT4 promoter transgene in mice, and this decrease is readily reversed by insulin administration

\section{Table 4}

Echocardiographic parameters in CIRKO and wild-type mice

\begin{tabular}{lcc}
\hline & Wild-type $(7)$ & CIRKO (14) \\
Parameter & $0.29 \pm 0.02$ & $0.32 \pm 0.01$ \\
LV diastolic dimension $(\mathrm{cm})$ & $0.12 \pm 0.022$ & $0.18 \pm 0.01^{\mathrm{A}}$ \\
LV systolic dimension $(\mathrm{cm})$ & $0.123 \pm 0.008$ & $0.096 \pm 0.004^{\mathrm{A}}$ \\
Intraventricular septum dimension $(\mathrm{cm})$ & $0.116 \pm 0.006$ & $0.093 \pm 0.004^{\mathrm{A}}$ \\
Posterior wall thickness $(\mathrm{cm})$ & $61.03 \pm 6.01$ & $43.34 \pm 2.69^{\mathrm{A}}$ \\
Fractional shortening & $0.91 \pm 0.04$ & $0.80 \pm 0.02^{\mathrm{A}}$ \\
Ejection fraction & $133 \pm 12$ & $107 \pm 5^{\mathrm{A}}$ \\
LV mass (mg) & $4.298 \pm 0.288$ & $3.635 \pm 0.157^{\mathrm{A}}$ \\
LV mass/body weight &
\end{tabular}

$A P<0.02$ vs. wild-type. Numbers of mice per group are in parentheses. 

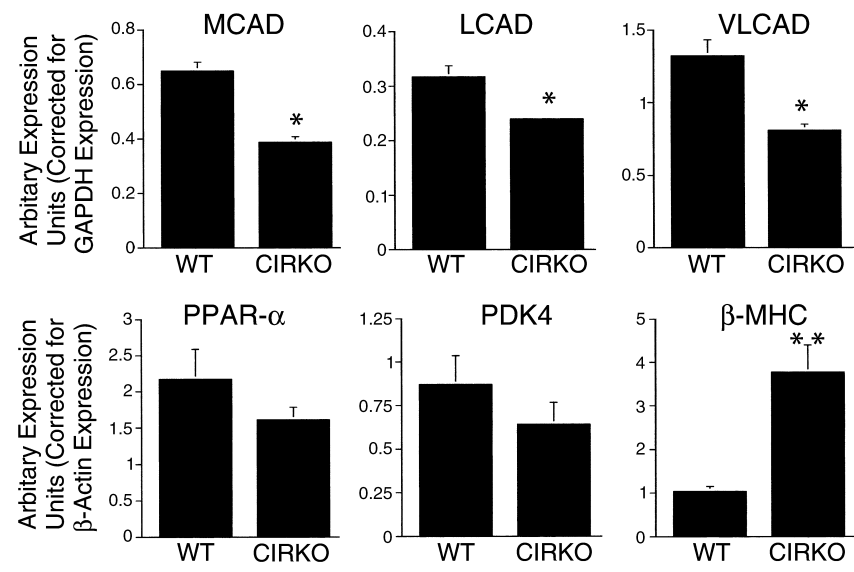

(23). By contrast, our finding of an increase in the abundance of GLUT4 proteins in the hearts of CIRKO mice suggests that deficient insulin signaling is not responsible for the reduction in GLUT4 levels in the heart in diabetes. Taken together, these data suggest that the changes in GLUT4 expression previously noted in models of insulin-deficient diabetes may be due to the rapid induction of hyperglycemia or other systemic metabolic effects and their reversal by insulin, as opposed to a direct effect of insulin on GLUT4 promoter activity per se. The CIRKO mouse is therefore an important model with which to further study the mechanisms by which GLUT4 expression in the heart is regulated in vivo.

The observations regarding diminished basal glucose uptake in isolated CIRKO cardiomyocytes, and the divergent evidence for increased glucose uptake in vivo or in working hearts, shed important insight into the relative roles of GLUT1 and GLUT4 in the regulation of cardiac glucose uptake. In noncontracting cardiomyocytes, downregulation of GLUT1 expression in CIRKO mice is associated with a proportionate reduction in basal cardiac glucose uptake. In contrast, in the intact heart in vivo and in isolated working hearts, there is clear evidence that glucose uptake and glycolysis are increased despite a reduction in GLUT1 expression. We believe that the basis for increased glucose uptake in CIRKO hearts is increased translocation of the expanded GLUT4 pool, stimulated in part by cardiac contraction. These observations are supported by similar findings in transgenic mice with increased cardiac expression of GLUT4 (10). Furthermore, in mice with cardiac-restricted GLUT4 deletion, studied after an overnight fast, cardiac glucose uptake is markedly reduced, despite a threefold increase in GLUT1 expression (55). Thus, although GLUT1 may be the major mediator of basal cardiac glucose uptake in quiescent cardiomyocytes, GLUT4 may be a more important regulator of glucose uptake in the contracting heart.

The reduced rates of glucose oxidation in CIRKO mice perfused without insulin are similar to reduced rates of glucose oxidation observed in the hearts of diabetic animals (21). Thus the possibility exists that

\section{Figure 7}

Gene expression analysis in CIRKO hearts. mRNA levels of MCAD, LCAD, VLCAD, PPAR $\alpha$, PDK 4 , and $\beta-M H C$ in hearts of WT and CIRKO mice. Real-time PCR data were obtained from four CIRKO and four WT littermate controls, and for Northern blot analysis from three CIRKO and three WT. Each transcript was analyzed in duplicate. Data are means \pm SE. ${ }^{*} P<0.05,{ }^{*} P<0.0007$ vs. WT.

diminished insulin signaling in the myocardium is an underlying mechanism for reduced glucose oxidation rates in the heart in diabetes. Diminished activity of the PDH complex, mediated in part by increased expression of PDK4, is one mechanism that has been implicated in diabetes (37). PDK4 expression was not increased in the hearts of CIRKO mice. We did not directly measure the activity of PDH in the present study; thus, reduced activity of PDH cannot be ruled out in this model. The increase in myocardial glucose oxidation observed after insulin stimulation was unexpected and indicates, for the first time to our knowledge, that the ability of insulin to increase glucose oxidation in heart muscle might not be mediated by insulin receptors in the cardiomyocyte. Insulin receptors are present in the vascular endothelium (56), and insulin has been shown to be a potent vasodilator of the coronary vascular bed (16). Indeed, coronary flow was significantly higher in insulin-treated CIRKO animals than in controls. Although it is unlikely that increased coronary flow can account for the increase in glucose oxidation, it is possible that mediators such as nitric oxide, which can be released from endothelial cells following insulin stimulation, may stimulate glucose oxidation (56). This is supported by observations that nitric oxide increases glucose uptake and glucose oxidation rates in isolated rat soleus muscle (57). In isolated cardiac myocytes, absolute rates of glucose uptake following treatment with $10 \mathrm{nM}$ IGF-1 were similar in CIRKO and wild-type myocytes. Thus the possibility also remains that the unexpected increase in glucose oxidation rates in CIRKO hearts perfused with $1 \mathrm{nM}$ insulin reflects signaling via IGF-1 receptors.

Goodwin et al. demonstrated that the glucose moieties destined to be oxidized in the heart preferentially arise from breakdown of glycogen (58). Thus, increased glycogen content in non-insulin-stimulated CIRKO hearts may be secondary to lower basal rates of glucose oxidation. The subsequent decline with insulin administration may reflect mobilization of glycogen in the face of the insulin-stimulated increase in glucose oxidation. Nitric oxide also regulates glycogen metabolism in skeletal muscle and liver by inhibiting glycogen synthesis and stimulating glycogenolysis $(59,60)$. Thus it is possible that this mechanism plays a role in decreasing the glycogen content in insulin-stimulated CIRKO hearts. In normal hearts, this effect is masked because the direct effect of insulin signaling in the myocardium increases glycogen synthesis that would counterbal- 
ance the nitric oxide-mediated effect. Future studies in this and other models, such as mice with absent insulin signaling in endothelial cells or other cell types such as smooth muscle cells, should provide additional mechanistic insight into insulin's ability to regulate cardiac myocyte glucose metabolism in a paracrine fashion.

Cardiac function was mildly reduced in isolated CIRKO hearts ex vivo and in vivo. It is possible that the reduced rates of fatty acid oxidation in CIRKO mice might lead to reduced production of ATP and phosphocreatine, which are not offset by increased glycolytic ATP production. The methodologies used in this study do not allow for direct measurements of high-energy phosphates in the heart. This issue can be addressed more directly in future studies using NMR spectroscopy, which would also allow for an analysis of the role of alternate substrates such as ketone bodies and lactate in the metabolic phenotype of CIRKO mice. It is likely that reduced cardiac function in vivo is at least partly the result of diminished cardiac mass, as evidenced by preserved contractile function of CIRKO cardiomyocytes in the absence of loading (61).

In summary, we have shown that insulin signaling is a critical determinant of adult cardiac size and plays an important developmental role in the regulation of myosin gene expression and substrate preference by the heart. Thus, insulin signaling may represent an important link between cardiac substrate utilization and the expression of genes that determine energy generation and energy consumption.

\section{Acknowledgments}

We would like to thank Dionne Rudder, Mikkael Yefremashvili, Timothy Barrette, and Deborah L. Jones for technical assistance. This work was supported by NIH grants DK-43526 (to D.A. McClain); HL-52338 (to S.E. Litwin); HL-43133 and HL/AG-61483 (to H. Taegtmeyer); DK-31036 and DK-33201 (to C.R. Kahn); and HL-62886, DK-02495, and HL-58073 (to E.D. Abel). S. Betuing was supported by a fellowship from the Association Française pour la Recherche Thérapeutique. S.E. Litwin is the recipient of a Merit Award from the Veterans Administration, and E.D. Abel is the recipient of a Research Award from the American Diabetes Association. Support of the Ben and Iris Margolis Foundation to the Utah Diabetes Center is also acknowledged.

1. Patti, M.E., and Kahn, C.R. 1998. The insulin receptor: a critical link in glucose homeostasis and insulin action. J. Basic Clin. Physiol. Pharmacol. 9:89-109.

2. Bruning, J.C., et al. 2000. Role of brain insulin receptor in control of body weight and reproduction. Science. 289:2122-2125.

3. Drummond-Barbosa, D., and Spradling, A.C. 2001. Stem cells and their progeny respond to nutritional changes during Drosophila oogenesis. Dev. Biol. 231:265-278.

4. Mihaylova, V.T., Borland, C.Z., Manjarrez, L., Stern, M.J., and Sun, H 1999. The PTEN tumor suppressor homolog in Caenorhabditis elegans regulates longevity and dauer formation in an insulin receptor-like signaling pathway. Proc. Natl. Acad. Sci. USA. 96:7427-7432.

5. Pierce, S.B., et al. 2001. Regulation of DAF-2 receptor signaling by human insulin and ins-1, a member of the unusually large and diverse $C$. elegans insulin gene family. Genes Dev. 15:672-686.

6. Bohni, R., et al. 1999. Autonomous control of cell and organ size by CHICO, a Drosophila homolog of vertebrate IRS1-4. Cell. 97:865-875.
7. Araki, E., et al. 1994. Alternative pathway of insulin signalling in mice with targeted disruption of the IRS-1 gene. Nature. 372:186-190.

8. Abel, E.D., et al. 1999. Cardiac hypertrophy with preserved contractile function after selective deletion of GLUT4 from the heart. J. Clin. Invest. 104:1703-1714

9. Depre, C., Rider, M.H., and Hue, L. 1998. Mechanisms of control of heart glycolysis. Eur. J. Biochem. 258:277-290.

10. Belke, D.D., Larsen, T.S., Gibbs, E.M., and Severson, D.L. 2001. Glucose metabolism in perfused mouse hearts overexpressing human GLUT-4 glucose transporter. Am. J. Physiol. Endocrinol. Metab. 280:E420-E427.

11. Laughlin, M.R., Taylor, J.F., Chesnick, A.S., and Balaban, R.S. 1992. Regulation of glycogen metabolism in canine myocardium: effects of insulin and epinephrine in vivo. Am. J. Physiol. 262:E875-E883.

12. Flaim, K.E., et al. 1983. Insulin effects on protein synthesis are independent of glucose and energy metabolism. Am. J. Physiol. 245:C133-C143.

13. Ren, J., Sowers, J.R., Walsh, M.F., and Brown, R.A. 2000. Reduced contractile response to insulin and IGF-I in ventricular myocytes from genetically obese Zucker rats. Am. J. Physiol. Heart Circ. Physiol. 279:H1708-H1714.

14. Aikawa, R., et al. 2000. Insulin prevents cardiomyocytes from oxidative stress-induced apoptosis through activation of PI3 kinase/Akt. Circulation. 102:2873-2879.

15. Baron, A.D. 1994. Hemodynamic actions of insulin. Am. J. Physiol. 267:E187-E202.

16. Jagasia, D., Whiting, J.M., Concato, J., Pfau, S., and McNulty, P.H. 2001 Effect of non-insulin-dependent diabetes mellitus on myocardial insulin responsiveness in patients with ischemic heart disease. Circulation. 103:1734-1739.

17. DeFronzo, R.A. 1981. The effect of insulin on renal sodium metabolism A review with clinical implications. Diabetologia. 21:165-171.

18. Brownsey, R.W., Boone, A.N., and Allard, M.F. 1997. Actions of insulin on the mammalian heart: metabolism, pathology and biochemical mechanisms. Cardiovasc. Res. 34:3-24.

19. Awan, M.M., and Saggerson, E.D. 1993. Malonyl-CoA metabolism in cardiac myocytes and its relevance to the control of fatty acid oxidation. Biochem. J. 295:61-66.

20. Chatham, J.C., Gao, Z.P., Bonen, A., and Forder, J.R. 1999. Preferential inhibition of lactate oxidation relative to glucose oxidation in the rat heart following diabetes. Cardiovasc. Res. 43:96-106.

21. Belke, D.D., Larsen, T.S., Gibbs, E.M., and Severson, D.L. 2000. Altered metabolism causes cardiac dysfunction in perfused hearts from diabetic (db/db) mice. Am. J. Physiol. Endocrinol. Metab. 279:E1104-E1113.

22. Chen, V., Ianuzzo, C.D., Fong, B.C., and Spitzer, J.J. 1984. The effects of acute and chronic diabetes on myocardial metabolism in rats. Diabetes. 33:1078-1084.

23. Olson, A.L., and Pessin, J.E. 1995. Transcriptional regulation of the human GLUT4 gene promoter in diabetic transgenic mice. J. Biol. Chem. 270:23491-23495.

24. Kolter, T., Uphues, I., and Eckel, J. 1997. Molecular analysis of insulin resistance in isolated ventricular cardiomyocytes of obese Zucker rats. Am. J. Physiol. 273:E59-E67.

25. Chatham, J.C. 2001. The impact of type 2 diabetes on the regulation of cardiac energy metabolism and function. J. Mol. Cell. Cardiol. 33:A153. (Abstr.)

26. Bruning, J.C., et al. 1998. A muscle-specific insulin receptor knockout exhibits features of the metabolic syndrome of NIDDM without altering glucose tolerance. Mol. Cell. 2:559-569.

27. Belke, D.D., Larsen, T.S., Lopaschuk, G.D., and Severson, D.L. 1999. Glucose and fatty acid metabolism in the isolated working mouse heart. $A m$. J. Physiol. 277:R1210-R1217.

28. Larsen, T.S., et al. 1999. The isolated working mouse heart: methodological considerations. Pflugers Arch. 437:979-985.

29. Cooksey, R.C., et al. 1999. Mechanism of hexosamine-induced insulin resistance in transgenic mice overexpressing glutamine:fructose-6-phosphate amidotransferase: decreased glucose transporter GLUT4 translocation and reversal by treatment with thiazolidinedione. Endocrinology. 140:1151-1157.

30. Chan, T.M., and Exton, J.H. 1976. A rapid method for the determination of glycogen content and radioactivity in small quantities of tissue or isolated hepatocytes. Anal. Biochem. 71:96-105.

31. Storlien, L.H., et al. 1991. Influence of dietary fat composition on development of insulin resistance in rats. Relationship to muscle triglyceride and omega-3 fatty acids in muscle phospholipid. Diabetes. 40:280-289.

32. Chomczynski, P., and Sacchi, N. 1987. Single-step method of RNA isolation by acid guanidinium thiocyanate-phenol-chloroform extraction. Anal. Biochem. 162:156-159.

33. Depre, C., et al. 1998. Unloaded heart in vivo replicates fetal gene expression of cardiac hypertrophy. Nat. Med. 4:1269-1275.

34. Manning, W.J., Wei, J.Y., Katz, S.E., Litwin, S.E., and Douglas, P.S. 1994 In vivo assessment of LV mass in mice using high-frequency cardiac ultrasound: necropsy validation. Am. J. Physiol. 266:H1672-H1675. 
35. Sack, M.N., et al. 1996. Fatty acid oxidation enzyme gene expression is downregulated in the failing heart. Circulation. 94:2837-2842.

36. Barger, P.M., and Kelly, D.P. 2000. PPAR signaling in the control of cardiac energy metabolism. Trends Cardiovasc. Med. 10:238-245.

37. Wu, P., et al. 1998. Starvation and diabetes increase the amount of pyruvate dehydrogenase kinase isoenzyme 4 in rat heart. Biochem. J. 329:197-201.

38. Mahdavi, V., Izumo, S., and Nadal-Ginard, B. 1987. Developmental and hormonal regulation of sarcomeric myosin heavy chain gene family. Circ. Res. 60:804-814.

39. Molkentin, J., and Dorn, G.W. 2001. Cytoplasmic signaling pathways that regulate cardiac hypertrophy. Annu. Rev. Physiol. 63:391-426.

40. MacLellan, W.R., and Schneider, M.D. 2000. Genetic dissection of cardiac growth control pathways. Annu. Rev. Physiol. 62:289-319.

41. Liu, J.P., Baker, J., Perkins, A.S., Robertson, E.J., and Efstratiadis, A. 1993. Mice carrying null mutations of the genes encoding insulin-like growth factor I (Igf-1) and type 1 IGF receptor (Igf1r). Cell. 75:59-72.

42. Burks, D.J., et al. 2000. IRS-2 pathways integrate female reproduction and energy homeostasis. Nature. 407:377-382.

43. Shioi, T., et al. 2000. The conserved phosphoinositide 3-kinase pathway determines heart size in mice. EMBOJ. 19:2537-2548.

44. Sack, M.N., Disch, D.L., Rockman, H.A., and Kelly, D.P. 1997. A role for $\mathrm{Sp}$ and nuclear receptor transcription factors in a cardiac hypertrophic growth program. Proc. Natl. Acad. Sci. USA. 94:6438-6443.

45. Vaulont, S., Vasseur-Cognet, M., and Kahn, A. 2000. Glucose regulation of gene transcription. J. Biol. Chem. 275:31555-31558.

46. Izumo, S., et al. 1987. Myosin heavy chain messenger RNA and protein isoform transitions during cardiac hypertrophy. Interaction between hemodynamic and thyroid hormone-induced signals. J. Clin. Invest. 79:970-977.

47. Depre, C., et al. 2000. Streptozotocin-induced changes in cardiac gene expression in the absence of severe contractile dysfunction. J. Mol. Cell. Cardiol. 32:985-996.

48. Young, M.E., Guthrie, P., Stepkowski, S.M., and Taegtmeyer, H. 2001. Glucose regulation of sarcomeric protein gene expression in the rat heart. $J$. Mol. Cell. Cardiol. 33:A181. (Abstr.)

49. Taegtmeyer, H., Hems, R., and Krebs, H.A. 1980. Utilization of energy- providing substrates in the isolated working rat heart. Biochem. J. 186:701-711.

50. Slieker, L.J., et al. 1992. Glucose transporter levels in tissues of spontaneously diabetic Zucker fa/fa rat (ZDF/drt) and viable yellow mouse (Avy/a). Diabetes. 41:187-193.

51. Kainulainen, H., et al. 1994. In vivo glucose uptake and glucose transporter proteins GLUT1 and GLUT4 in heart and various types of skeletal muscle from streptozotocin- diabetic rats. Biochim. Biophys. Acta. 1225:275-282.

52. Kraegen, E.W., et al. 1993. Glucose transporters and in vivo glucose uptake in skeletal and cardiac muscle: fasting, insulin stimulation and immunoisolation studies of GLUT1 and GLUT4. Biochem. J. 295:287-293.

53. Laybutt, D.R., Thompson, A.L., Cooney, G.J., and Kraegen, E.W. 1997. Selective chronic regulation of GLUT1 and GLUT4 content by insulin, glucose, and lipid in rat cardiac muscle in vivo. Am. J. Physiol. 273:H1309-H1316

54. Montessuit, C., and Thorburn, A. 1999. Transcriptional activation of the glucose transporter GLUT1 in ventricular cardiac myocytes by hypertrophic agonists. J. Biol. Chem. 274:9006-9012.

55. Tian, R., and Abel, E.D. 2001. Responses of GLUT4-deficient hearts to ischemia underscore the importance of glycolysis. Circulation. 103:2961-2966.

56. Zeng, G., et al. 2000. Roles for insulin receptor, PI3-kinase, and Akt in insulin-signaling pathways related to production of nitric oxide in human vascular endothelial cells. Circulation. 101:1539-1545.

57. Young, M.E., Radda, G.K., and Leighton, B. 1997. Nitric oxide stimulates glucose transport and metabolism in rat skeletal muscle in vitro. Biochem. J. 322:223-228.

58. Goodwin, G.W., Ahmad, F., Doenst, T., and Taegtmeyer, H. 1998. Energy provision from glycogen, glucose, and fatty acids on adrenergic stimulation of isolated working rat hearts. Am. J. Physiol. 274:H1239-H1247.

59. Borgs, M., et al. 1996. Modulation of basal hepatic glycogenolysis by nitric oxide. Hepatology. 23:1564-1571.

60. Sprangers, F., et al. 1998. Nitric oxide inhibits glycogen synthesis in isolated rat hepatocytes. Biochem. J. 330:1045-1049.

61. Litwin, S.E., Zhang, D., Graveleau, C., and Abel, E.D. 2001. Impact of cardiac selective ablation of the insulin receptor on myocyte mechanics and in vivo cardiac contractility. Circulation. 104(Suppl. II):II-569. (Abstr.). 